JL Jane G. Gatela, MD

Department of Otorhinolaryngology Head and Neck Surgery East Avenue Medical Center
Correspondence: Dr. JL Jane G. Gatela Department of Otorhinolaryngology Head and Neck Surgery

6th Floor, East Avenue Medical Center

East Avenue, Diliman, Quezon City 1100 Philippines

Phone: (632) 89280611 local 324/ (632) 9062304704

Email: eamc_enthns@yahoo.com jljane.gatela@gmail.com

The author declared that this represents original material that is not being considered for publication or has not been published or accepted for publication elsewhere in full or in part, in print or electronic media; that the manuscript has been read and approved by the author, that the requirements for authorship have been met by the author, and that the author believe that the manuscript represents honest work.

Disclosures: The author signed disclosures that there are no financial or other (including personal) relationships, intellectual passion, political or religious beliefs, and institutional affiliations that might lead to a conflict of interest.

Presented at the Philippine Society of Otolaryngology-Head and Neck Surgery 1st Virtual COVID-19 Research Forum. November 18, 2020

\title{
Self-Inflicted Craniofacial Impalement Injury with a Screwdriver During the COVID-19 Pandemic: A Case Report
}

\begin{abstract}
Objective: To present a case of a 37-year-old man presenting with craniofacial impalement injury from a screwdriver that happened during the early stages of the COVID-19 pandemic.
\end{abstract}

\section{Methods:}

$\begin{array}{ll}\text { Design: } & \text { Case Report } \\ \text { Setting: } & \text { Tertiary Government Training Hospital } \\ \text { Patient: } & \text { One }\end{array}$

Results: During the early stages of COVID-19 pandemic a 37-year-old man was brought to the emergency room with a screwdriver embedded in his right eye. A multidisciplinary team observing available recommendations (level IV PPE, carefully planned operative directives) successfully performed endoscopic endonasal transsphenoidal surgery with application of a nasoseptal Hadad flap and abdominal fat obliteration. Aside from medial gaze limitations of the right eye, there was no CSF leak or rhinorrhea and no neurologic sequelae on follow up.

Conclusion: Endoscopic skull base surgery for such an impalement injury as this is a formidable multidisciplinary challenge, even in normal times. The early stages of the COVID-19 pandemic presented additional challenges. Observing evolving guidelines minimized the high risk of exposure for health care workers while maximizing care for the patient.

\section{Keywords: craniofacial impalement injuries; endoscopic sinus surgery; COVID-19 pandemic}

Craniofacial impalement injuries are uncommon, and when encountered are a cause for conversation because of their eccentric location and strange objects involved. ${ }^{1}$ Skull base involvement makes cases even more complex, potentially causing such complications as CSF leak, meningitis, hydrocephalus, nerve or vessel injury, and even death. ${ }^{2}$ Even in the best of times, the skull base surgery required for such an impalement injury poses a formidable multidisciplinary challenge. However, this case presented in April 2020, during the early surge of the COVID-19 pandemic. We present our experience in managing this case while trying to observe evolving guidelines to minimize risks of exposure for health care workers while maximizing care for the patient. 


\section{CASE REPORT}

A 37-year-old man who was found lying unconscious at home with a screwdriver embedded in his right eye was brought to the emergency room by a relative. The patient had been observed to be in a depressed mood over the previous weeks, self-inflicting wounds on his wrist. On later review of history, the patient claimed to have no recall of events preceding the incident. He also claimed to have been hearing voices accusing him of doing something he did not do and forcing him to harm himself.

He arrived at the emergency room with Glasgow Coma Score (GCS) 13 (E3V4M6), was awake, ambulatory, opened eyes on verbal command, and was conversant but disoriented to time and place, confused, and had an object impaled in the medial canthus of the right eye. (Figure 1A, B) Ophthalmologic evaluation revealed an intact globe with visual acuity of counting fingers on the right and $6 / 6$ on the left, right extraocular muscle movement limitation (unable to move horizontally), with retinal edema but no papilledema on fundoscopy. The right upper and lower canaliculi were transected. Psychiatric assessment highly considered schizophrenia and Risperidone $2 \mathrm{mg} /$ tablet, $1 \frac{2}{2}$ tablet was started once daily.

A plain cranial CT scan with orbital cuts showed a high attenuation metallic density traversing the medial aspect of the right orbit apparently in the intra orbital compartment with suspicious discontinuity of the right medial rectus muscle, through the sphenoid sinus, pons and probably cerebellar tonsils also. There were subtle hyperdensities (suggestive of intraparenchymal hemorrhage) in the pons and anterior aspect of the right cerebellum. There were fractures of the right lamina papyracea and anterior and posterior walls of the right sphenoid sinus. (Figure 2A, B)

Prophylactic intravenous antibiotics (ceftriaxone 2grams once a day, metronidazole $500 \mathrm{mg}$ every 8 hours and oxacillin $500 \mathrm{mg}$ every 6 hours) were started and removal of the foreign object through a combined direct and endonasal transsphenoidal approach with abdominal fat obliteration and a Hadad - Bassagasteguy flap, and lacrimal probing and medial canthal repair under general anesthesia were planned.

Because there were no clear surgical guidelines during the early stages of the COVID-19 pandemic, the procedure was delayed for 5 days to achieve adequate planning and to ensure the safety of the surgical team, staff and patient. Throughout this time, the patient was ambulatory, with stable vital signs, no sensorial changes and no progression of symptoms. He had no symptoms or signs of COVID-19 infection, and was cleared for the procedure by the infectious disease service without requiring an RT-PCR swab test.

Our patient was transported to the operating room (OR) theater through a dedicated path for non-COVID patients. The OR air conditioning had been turned off to lessen the chance of aerosol circulation (a negative pressure environment was not yet available in our institution during this time), and the theater had been sanitized. All instruments and equipment were prepared inside the $\mathrm{OR}$, sanitized
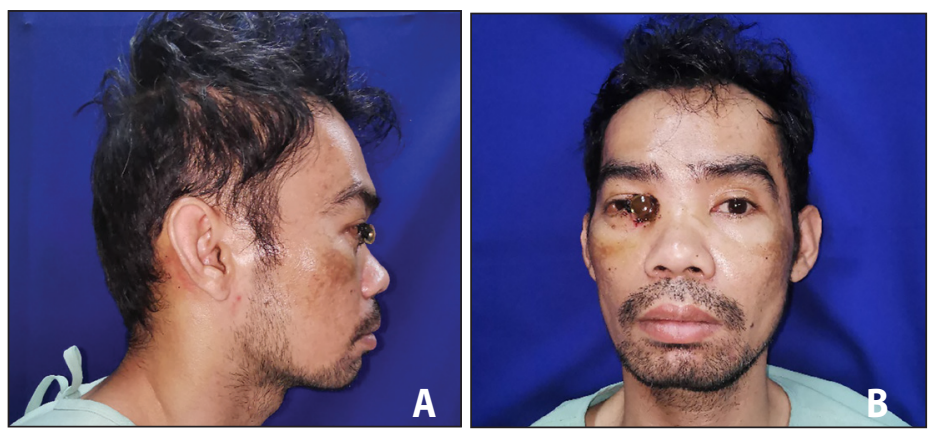

Figure 1. Preoperative photos A. lateral view; and B. frontal view showing embedded screwdriver in right medial canthus (photos published in full, with permission).

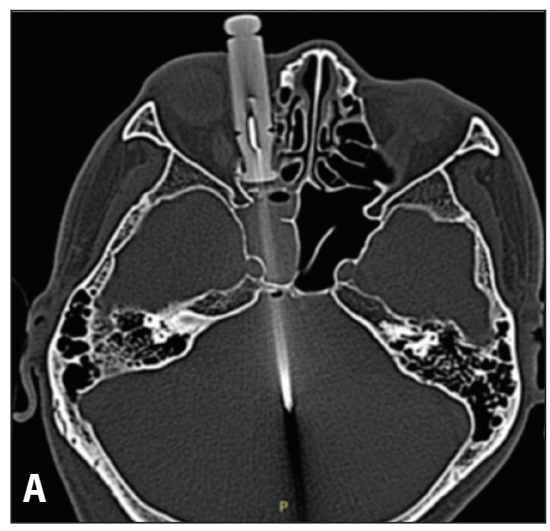

Figure 2. Plain Cranial CT scans A. Axial view; and B. sagittal view showing a high attenuation metallic density $(400$ $\mathrm{HU}$ ) traversing the medial aspect of the right orbit, sphenoid sinus, pons, and probably cerebellar tonsils. Note subtle hyperdensities (intraparenchymal hemorrhage) in the pons and anterio aspect of the right cerebellum, and fractures in the right lamina papyracea and anterior and posterior walls of the right sphenoid sinus.

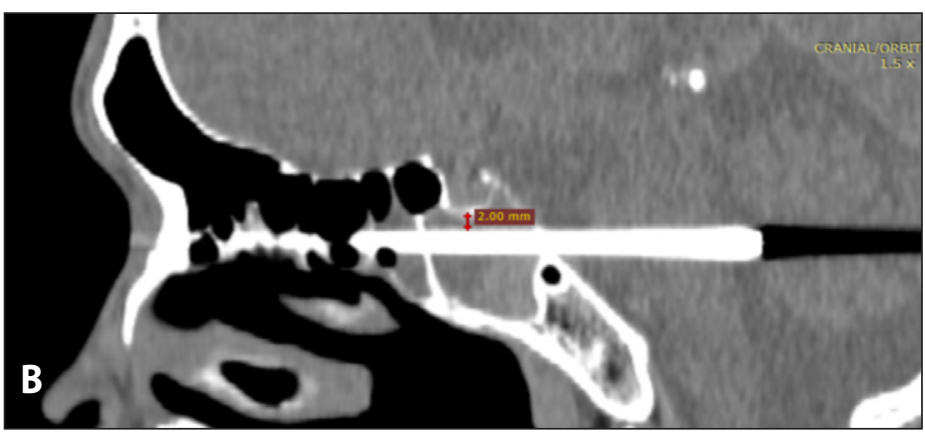

and well covered, eliminating the need to transit in and out of the room. Personnel were equipped with complete personal protective equipment (PPEs) including respiratory masks, head caps, double gloves, shoe covers and face shields. Proper donning and doffing areas were provided with designated containers for disposal of PPEs, linen and other materials. All nonessential staff left the room during intubation, returning only after the airway was secured (they later also left the room during extubation). Otherwise, the OR door was closed, with no ingress or egress during the entire surgery.

The operative procedure started with harvesting of a nasoseptal Hadad flap that was tucked into the nasopharynx. (Figure 3A, B) A 2x2 $\mathrm{cm}$ abdominal fat graft was harvested from the left lower abdominal quadrant. The anterior sphenoid ostium was widened and the metal shaft of the impaled object was visualized. (Figure $3 C$ ) The shaft was $2 \mathrm{~mm}$ below the sella turcica, using the vidian canal as a landmark. 


\section{CASE REPORTS}
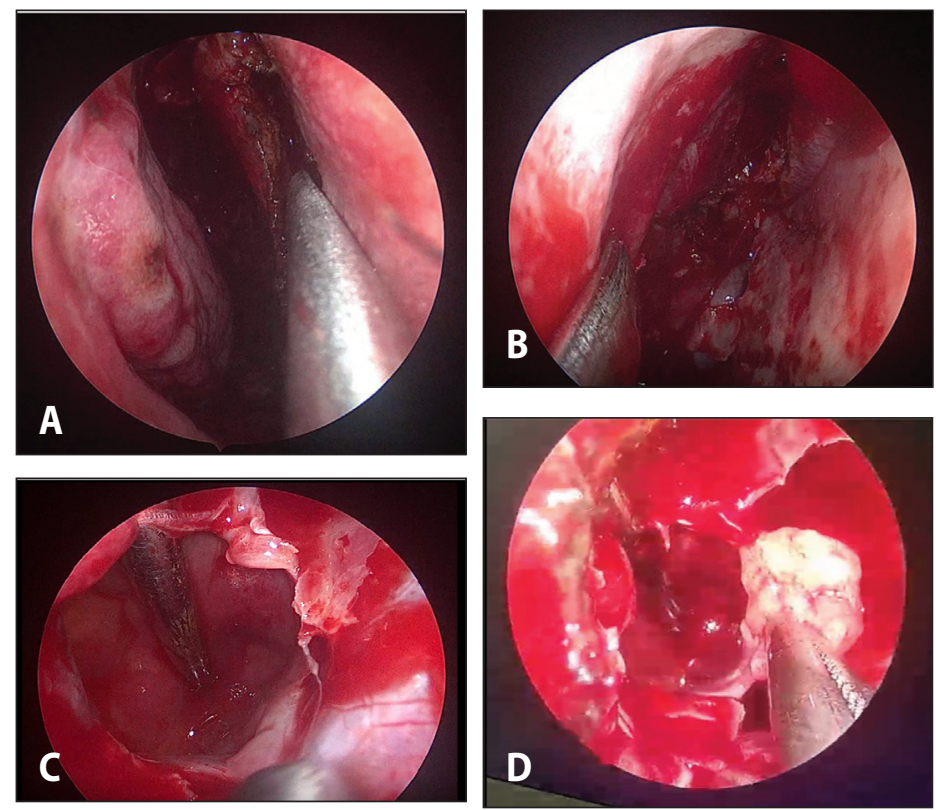

Figure 3. Operative procedure showing A. incision of nasoseptal flap; B. tucking in of nasoseptal flap into nasopharynx; C. visualization of foreign body through anterior sphenoid ostium; and D. abdominal fat graft laid over the post-extraction defect.

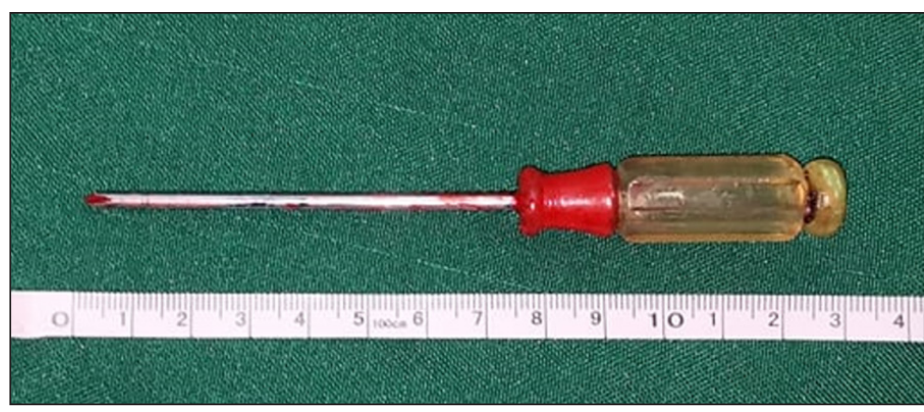

Figure 4. Post-extraction photo of $13 \mathrm{~cm}$ penetrating object (Screwdriver)
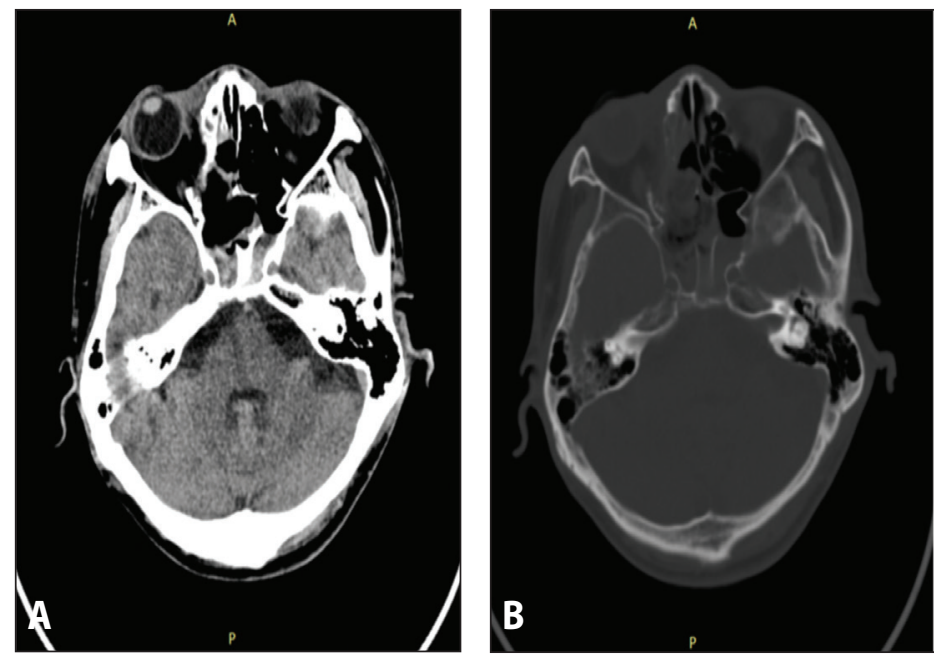

Figure 5. Postoperative CT scan images, axial views A. bone window; and B. cranial window. The previously noted metallic foreign body (screwdriver) is no longer seen.
Under endoscopic visualization of the sphenoid, the screwdriver was extracted externally, measuring $13 \mathrm{~cm}$ in length. (Figure 4) An abrupt cerebro-spinal fluid (CSF) leak from the $5 \mathrm{~mm}$ defect after the screwdriver extracted was controlled with an abdominal fat graft plug over which a composite middle turbinate graft composed of bone and mucosa was laid. (Figure 3D) Lastly, the nasoseptal flap was placed over the middle turbinate graft with the mucoperichondrial side facing the skull base, and a nonabsorbable nasal pack was placed. Ophthalmologic exploration with lacrimal probing in the right eye revealed transected upper and lower canaliculi, and primary closure was just done using PGA 6-0.

The entire procedure was completed in three hours, and PPEs were doffed and disposed in designated containers. All involved personnel showered after the procedure before donning outside clothes. All equipment and instruments were left in theater to be cleaned together.

There were no postoperative complications. The postoperative CT scan confirmed complete extraction of the foreign body. (Figure 5A, B)

The patient was discharged 2 weeks later after completion of antibiotics, and the patient and all personnel involved in this surgery were symptom-free of COVID-19 infection. There was no CSF leak or rhinorrhea following nasal pack removal on the 14th postoperative day. Risperidone was discontinued after unremarkable psychiatric evaluation, but the right medial gaze limitation and episodes of uncontrolled tearing of the right eye persisted at four months' follow-up. After 6 months, full extraocular movements were already appreciated. (Figure 6) Endoscopy showed a healed posterior sphenoid wall with a remnant of fat that was used to obliterate the defect. (Figure 7)

\section{DISCUSSION}

Craniofacial impalement injuries encountered in the emergency department are more often than not challenging, but they become even more challenging in the context of a surging COVID 19 pandemic. Craniofacial impalement injury seldom occurs and usually only a few survive when it happens because of its fatal complications. ${ }^{3}$

Pavlidis et al. discussed 21 incidents of perforating screwdriver craniocerebral injuries reported from 1950 to 2016, of which only 2 out of 21 were suicide attempts. ${ }^{4}$ Impalement in the orbital area is uncommon, accounting only for 2 out of the 21 cases, although the mortality rate for overall screwdriver impalement was high (9 out of 21 cases; 47.6\%). ${ }^{4}$ Fortunately, our patient was able to survive and recover.

Intracranial foreign body penetration (such as from a screwdriver) can cause such sudden complications as subarachnoidal or intraparenchymal cerebral hemorrhages, CSF rhinorrhea, and pneumocephalus as well as delayed severe complications like meningitis and cerebral abscess. ${ }^{5}$ Increased intracranial pressure from edema or cerebral hemorrhage can also crush sensitive cerebral tissue. ${ }^{4}$ Most deaths due to penetrating trauma are induced by the 

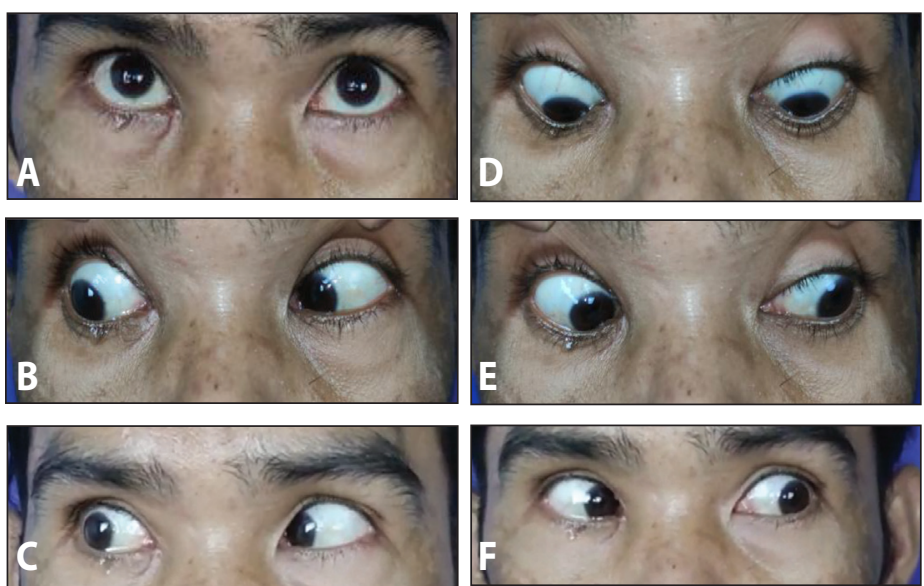

Figure 6. A-F. Gaze evaluation after 6 months showing full extraocular muscle movement A. elevation; B. dextrodepression; C. dextreversion; D. depression; E. levodepression; and F. levoversion.

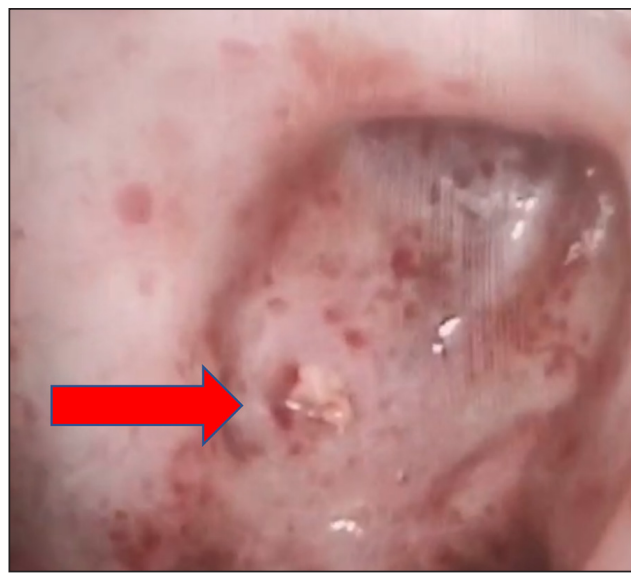

Figure 7. Endoscopic view of sphenoid sinus posterior wall after 6 months showing healed surface and fat remnant used to obliterate the defect site (arrow).

laceration of blood vessels. ${ }^{4}$ Resuscitation and stabilization of the patient are still the initial management of any craniofacial impalement injury. ${ }^{5}$

According to Kazim et al., surgical treatment should be performed within 12 hours of injury to decrease the risk of infectious complications such as meningitis or brain abscess. ${ }^{6}$ Delays greater than 48 hours from the time of injury increase the incidence of infection dramatically from $4.6 \%$ to $36.5 \%$ making antibiotic coverage necessary. ${ }^{6}$ It is recommended that broad spectrum antibiotics be instituted in all cases of penetrating brain injury, started as soon as possible and maintained for at least 7-14 days. ${ }^{6}$ In our case, prophylactic antibiotics were started while preparing for the surgery that took place 5 days after injury.

Our case took place on April 4, 2020 during the early days of the COVID-19 global pandemic. ${ }^{7}$ We knew then that the virus primarily infected the mucosa of the upper and lower airways with the highest viral load present in nasal tissue, placing otolaryngologists in particularly high risk situations for COVID-19 infection. ${ }^{8}$ Because COVID-19 was relatively new in the Philippines, there was no established protocol on handling trauma patients during this time when recommendations, experiences and expertise were being shared on social media and other channels of information. We had to decide how to manage our patient during the pandemic and minimize the risk of exposure for healthcare workers. There was only one operating room that was made available to all cutting services resulting in a long queue of emergency procedures. Moreover, endoscopic surgery and endotracheal intubation for general anesthesia were classified as high-risk aerosol generating procedures..$^{9,10}$ Other factors of concern at the time were extended duration of exposure, proximity to the airway, manipulation of high viral load tissue and aerosolization through the use of energy devices such as drills. Careful planning and coordination between the services involved (neurosurgery, otorhinolaryngology - head and neck surgery, ophthalmology, anesthesiology, nursing) was needed to facilitate a smooth and quick operative procedure, minimize movement in and out of the room, and minimize duration of exposure. Fortunately, we succeeded in achieving all these.

Indeed, craniofacial impalement injuries such as our case should be managed through a multidisciplinary approach. We succeeded in successful endoscopic endonasal removal of a $13 \mathrm{~cm}$ screwdriver that pierced through the medial canthus of right eye and penetrated through the sphenoid sinus into the cranium. The procedure was planned and executed in a careful manner, observing protective protocols against COVID-19 while maintaining the best possible level of care we could give our patient in our particular context. We hope our report encourages our colleagues and the healthcare community to continue striving and adapting to the new norm. Let us not lose hope in our continued pursuit of combating COVID-19.

\section{REFERENCES}

1. Eppley BL. Craniofacial impalement injury: a rake in the face. J Craniofac Surg. 2002 Jan;13(1):35 7. DOI: 10.1097/00001665-200201000-00006; PubMed PMID: 11886989.

2. Samuelson MB, Chandra RK, Russell PT, Weaver KD. Sinonasal metallic foreign body penetration of the anterior cranial fossa. Otolaryngology Case Reports. 2017 Feb 1;2:10-2. DOI: 10.1016/j. xocr.2017.02.002.

3. Nadeau DS, Hazzi C. Self-inflicted crossbow injury in an adult: challenges of surgical management with skull base disruption and airway precariousness. Journal of Otolaryngology Head and Neck Diseases. 2020 July 11:2(1). [cited 2020 July 11]: Available from: https:// escientificpublishers.com/self-inflicted-crossbow-injury-in-an-adult-challenges-of-surgicalmanagement-with-skull-base-disruption-and-airway-precariousness-JOHND-02-0012.

4. Pavlidis $P$, Karakasi MV, Birbilis TA. Traumatic brain injury due to screwdriver assaults: literature review and case report. Am J Forensic Med Pathol. 2016 Dec;37(4):291-298. DOI: 10.1097/ paf.0000000000000267 PubMed PMID: 27571172.

5. AS Y, Mahmud MR, Alfin JD, Adeleke NA. Clinical presentation and outcome of impalement craniocerebral injuries- a case series. J West Afr Coll Surg. 2017 Apr-Jun;7(2):112-123. PubMed PMID: 29951469; PubMed Central PMCID: PMC6016750.

6. Kazim SF, Shamim MS, Tahir MZ, Enam SA, Waheed S. Management of penetrating brain injury. J Emerg Trauma Shock. 2011 Jul-Sep;4(3):395-402. DOI: 10.4103/0974-2700.83871; PubMed PMID: 21887033; PubMed Central PMCID: PMC3162712.

7. Mohan BS, Nambiar V. COVID-19: An insight into SARS-CoV-2 pandemic originated at Wuhan City in Hubei Province of China. J Infect Dis Epidemiol. 2020;6(4):146. DOI: 10.23937/24743658/1510146.

8. Lammers MJW, Lea J, Westerberg BD. Guidance for otolaryngology health care worker performing aerosol generating medical procedures during the COVID-19 pandemic. $J$ Otolaryngol - Head Neck Surg. 2020 Jun 3;49(1):36 DOI: 10.1186/s40463-020-00429-2. PubMed.

9. Givi B, Schiff BA, Chinn SB, Clayburgh D, lyer NG, Jalisi S, et al. Safety recommendation for evaluation and surgery of the head and neck during the COVID-19 pandemic. JAMA Otolaryngol Head Neck Surg. 2020 Jun 1;146:579-584. DOI:10.1001/jamaoto.2020.0780 PubMed PMID: 32232423.

10. Howard BE. High-risk aerosol-generating procedures in COVID-19: respiratory protective equipment considerations. Otolaryngol Head Neck Surg. 2020 Jul;163(1):98-103. DOI: 10.1177/0194599820927335; PubMed PMID: 32396451 